\title{
Technical Note: A new SIze REsolved Aerosol Model (SIREAM)
}

\author{
E. Debry, K. Fahey, K. Sartelet, B. Sportisse, and M. Tombette \\ CEREA, Joint Research Laboratory, École Nationale des Ponts et Chaussées / EDF R\&D, France \\ Received: 9 November 2006 - Published in Atmos. Chem. Phys. Discuss.: 22 November 2006 \\ Revised: 26 February 2007 - Accepted: 2 March 2007 - Published: 21 March 2007
}

\begin{abstract}
We briefly present in this short paper a new SIze REsolved Aerosol Model (SIREAM) which simulates the evolution of atmospheric aerosol by solving the General Dynamic Equation (GDE). SIREAM segregates the aerosol size distribution into sections and solves the GDE by splitting coagulation and condensation/evaporationnucleation. A quasi-stationary sectional approach is used to describe the size distribution change due to condensation/evaporation, and a hybrid equilibrium/dynamical masstransfer method has been developed to lower the computational burden. SIREAM uses the same physical parameterizations as those used in the Modal Aerosol Model, MAM Sartelet et al. (2006). It is hosted in the modeling system Polyphemus Mallet et al., 2007, but can be linked to any other three-dimensional Chemistry-Transport Model.
\end{abstract}

\section{Introduction}

Atmospheric particulate matter (PM) has been negatively linked to a number of undesirable phenomena ranging from visibility reduction to adverse health effects. It also has a strong influence on the earth's energy balance Seinfeld and Pandis (1998). As a result, many governing bodies, especially in North America and Europe, have imposed increasingly stringent standards for PM. As an exemple the 1999/30/CE European Directive has imposed a daily PM $_{1} 0$ concentration limit of $50 \mu \mathrm{g} / \mathrm{m}^{3}$ since January 2005.

Atmospheric aerosol is a complex mixture of inorganic and organic components, with composition varying over the size range of a few nanometers to several micrometers. These particles can be emitted directly from various anthropogenic and biogenic sources or can be formed in the atmosphere by organic or inorganic precursor gases.

Given the complexity of PM, its negative effects, and the desire to control atmospheric PM concentrations, models that accurately describe the important processes that affect the aerosol size/composition distribution are therefore crucial. Three-dimensional Chemistry-Transport Mod-

Correspondence to: $\mathrm{B}$. Sportisse

(bruno.sportisse@cerea.enpc.fr) els (CTMs) provide the necessary tools to develop not only a better understanding of the formation and the distribution of PM but also sound strategies to control it. Historically, CTMs focused on ozone formation or acid deposition and did not include a detailed treatment of aerosol. Several models have been developed that include a very thorough treatment of aerosol processes Gong et al. (2003); Adams and Seinfeld (2002); Spracklen et al. (2005), but there are still many limitations Seigneur (2001).

In detailed models that seek to describe the time and spatial evolution of atmospheric PM, it is necessary to include those processes described in the General Dynamic Equation for aerosols (condensation/evaporation, coagulation, nucleation, inorganic and organic thermodynamics). These and additional processes like heterogeneous reactions at the aerosol surface, mass transfer between aerosol and cloud droplets, and aqueous-phase chemistry inside cloud droplets represent some of the most important mechanisms for altering the aerosol size/composition distribution.

Among the aerosol models, one usually distinguishes between modal models Whitby and McMurry (1997) and size resolved or sectional models Gelbard et al. (1980). We refer for instance to the modal model of Binkowski and Roselle (2003) and the sectional model of Zhang et al. (2004) for a description of state-of-the-science aerosol models, hosted by the Chemistry-Transport Model, CMAQ Byun and Schere (2004).

Here we present the development of a new SIze REsolved Aerosol Model (SIREAM). SIREAM is the sizeresolved alternative to the modal model, MAM Modal Aerosol Model, Sartelet et al. (2006). Both models use the same physical parameterizations through the library for atmospheric physics and chemistry ATMODATA Mallet and Sportisse (2005). Both have a modular approach and rely on different model configurations. They are hosted in the modeling system POLYPHEMUS Mallet et al., 2007 and used in several global, regional and local eulerian applications. A detailed description of SIREAM and MAM can be found in Sportisse et al. (2006) (available at http://www.enpc.fr/cerea/ polyphemus). A key feature of SIREAM is its modular design, as opposed to an all-in-one model. SIREAM can be

Published by Copernicus GmbH on behalf of the European Geosciences Union. 
used in many configurations and is intended for ensemble modeling (similar to Mallet and Sportisse (2006)).

This paper is structured as follows. The model formulation and main parameterizations included in SIREAM are described in Sect. 2. The numerical algorithms used for solving the GDE are given in Sect. 3. A specific focus is devoted to condensation/evaporation, which is by far the most challenging issue.

\section{Model formulation}

In this section we focus on aerosol dynamics, i.e. on the nucleation, condensation/evaporation, and coagulation processes. In addition, we briefly describe some processes that are strongly related to aerosols (heterogeneous reactions at the aerosol surface, mass transfer between the aerosols and the cloud droplets and aqueous-phase chemistry in cloud droplets). We also include the parameterizations for SemiVolatile Organic Compounds (SVOCs).

In order to deal with different parameterizations and to avoid the development of an "all-in-one" model, the parameterizations have been implemented as functions of the library ATMoDATA (Mallet and Sportisse (2005)), a package for atmospheric physics. As such, they can be used by other models.

\subsection{Composition}

The particles are assumed to be internally mixed, i.e., that there is a unique chemical composition for a given size. Each aerosol may be composed of the following components :

- liquid water;

- inert species : mineral dust, elemental carbon and, in some applications, heavy trace metals (lead, cadmium) or radionuclides bound to aerosols;

- inorganic species : $\mathrm{Na}+, \mathrm{SO}_{4}^{2-}, \mathrm{NH}_{4}+, \mathrm{NO}_{3}^{-}$and $\mathrm{Cl}^{-}$;

- organic species : one species for Primary Organic Aerosol (POA), 8 species for Secondary Organic Aerosol (see below for more details).

A typical version of the model (trace metals or radionuclides are not included) tracks the evolution of 17 chemical species for a given size bin $(1+2+5+1+8)$. These species (external species) should be distinguished from the species that are actually inside one aerosol in different forms (ionic, dissolved, solid). Let $n_{e}$ be the number of external species.

The internal composition for inorganic species is determined by thermodynamic equilibrium, solved by ISORROPIA v.1.7 Nenes et al. (1998). Water is assumed to quickly reach equilibrium between the gas and aerosol phases. Its concentration is given by the thermodynamic model (through the Zdanovskii-Stokes-Robinson relation).
The organic composition is given by the SORGAM model Schell et al. (2001b) which we detail in Sect. 2.2.5.

Hereafter, the particle mass $m$ refers to the dry mass. In order to reduce the wide range of magnitude over the particle size distribution and to better represent small particles, the particle distribution is described with respect to the logarithmic mass $x=\ln m$ Wexler et al. (1994); Meng et al. (1998); Gaydos et al. (2003).

The particles are described by a number distribution, $n(x, t)\left(\right.$ in $\left.^{-3}\right)$, and by the mass distributions for species $\mathrm{X}_{\mathrm{i}}$, $\left\{q_{i}(x, t)\right\}_{i=1, n_{e}}\left(\right.$ in $\left.\mu \mathrm{g} \cdot \mathrm{m}^{-3}\right)$. The mass distributions satisfy $\sum_{i=1}^{i=n_{e}} q_{i}=m n$. We also define the mass $m_{i}(x, t)=\frac{q_{i}(x, t)}{n(x, t)}$ of species $\mathrm{X}_{\mathrm{i}}$ in the particle of logarithmic mass $x$. It satisfies $\sum_{i=1}^{i=n_{e}} m_{i}(x, t)=e^{x}$.

\subsection{Processes and parameterizations for the GDE}

\subsubsection{Nucleation}

The formation of the smallest particles is given by the aggregation of gaseous molecules leading to thermodynamically stable clusters. The mechanism is poorly known and most models assume homogeneous binary nucleation of sulfate and water to be the major mechanism in the formation of new particles. Binary schemes tend to underpredict nucleation rates in comparison with observed values. Korhonen et al. (2003) has indicated that for the conditions typical in the lower troposphere ternary nucleation of sulfate, ammonium and water may be the only relevant mechanism.

SIREAM offers two options for nucleation: the $\mathrm{H}_{2} \mathrm{O}-$ $\mathrm{H}_{2} \mathrm{O}_{4}$ binary nucleation scheme of Vehkamki et al. (2002) and the $\mathrm{H}_{2} \mathrm{O}-\mathrm{H}_{2} \mathrm{O}_{4}-\mathrm{NH}_{3}$ ternary nucleation scheme of Napari et al. (2002).

The output is a nucleation rate, $J_{0}$, a nucleation diameter, and chemical composition for the nucleated particles. The new particles are added to the smallest bin.

\subsubsection{Coagulation}

Atmospheric particles may collide with one another due to their Brownian motion or due to other forces (e.g., hydrodynamic, electrical or gravitational). SIREAM includes a description of Brownian coagulation, the dominant mechanism in the atmosphere. There may be a limited effect on the particle mass distribution and this process is usually neglected Zhang et al. (2004). However coagulation may have substantial impact on the number size distribution for ultrafine particles.

The coagulation kernel $K(x, y)$ (in unit of volume per unit of time) describes the rate of coagulation between two particles of dry logarithmic masses $x$ and $y . K$ has different expressions depending on the relevant regime Seinfeld and Pandis (1998). 


\subsubsection{Condensation/evaporation}

Some gas-phase species with a low saturation vapor pressure may condense on existing particles while some species in the particle phase may evaporate. The mass transfer is governed by the gradient between the gas-phase concentration and the concentration at the surface of the particle. The mass flux for volatile species $\mathrm{X}_{\mathrm{i}}$ between the gas phase and one particle of logarithmic mass $x$ is computed by:

$$
\frac{d m_{i}}{d t}=I_{i}=2 \pi D_{i}^{g} d_{p} f_{F S}\left(K_{n_{i}}, \alpha_{i}\right)\left(c_{i}^{g}-c_{i}^{s}(x, t)\right)
$$

$d_{p}$ is the particle wet diameter (see Sect. 2.2.6 for the relation to mass). $D_{i}^{g}$ and $c_{i}^{g}$ are the molecular diffusivity in the air and the gas-phase concentration of species $\mathrm{X}_{\mathrm{i}}$, respectively. The concentration $c_{i}^{s}$ at the particle surface is assumed to be at local thermodynamic equilibrium with the particle composition:

$c_{i}^{s}(x, t)=\eta\left(d_{p}\right) c_{i}^{e q}\left(q_{1}(x, t), \ldots, q_{n_{e}}(x, t), R H, T\right)$

$T$ is the temperature and $R H$ is the relative humidity. $\eta\left(d_{p}\right)=\exp \left(\frac{4 \sigma v_{p}}{R T d_{p}}\right)$ is a correction for the Kelvin effect, with $\sigma$ the surface tension, $R$ the gas constant and $v_{p}$ the particle molar volume. In practice, $c_{i}^{e q}$ is computed by the reverse mode of a thermodynamics package like ISORROPIA Nenes et al. (1998) in the case of kinetic mass transfer.

The Fuchs-Sutugin function, $f_{F S}$, describes the noncontinuous effects (Dahneke (1983)). It depends on the Knudsen number of species $\mathrm{X}_{\mathrm{i}}, K_{n_{i}}=\frac{2 \lambda_{i}}{d_{p}}$ (with $\lambda_{i}$ the air mean free path), and on the accommodation coefficient $\alpha_{i}$ (default value is 0.5 ):

$f_{F S}\left(K_{n_{i}}, \alpha_{i}\right)=\frac{1+K_{n_{i}}}{1+2 K_{n_{i}}\left(1+K_{n_{i}}\right) / \alpha_{i}}$

When particles are in a liquid state, the condensation of an acidic component may free hydrogen ions and the condensation of a basic component may consume hydrogen ions. Thus the condensation/evaporation (c/e hereafter) process may have an effect on the particle $p H$. The hydrogen ion flux induced by mass transfer is:

$J_{\mathrm{H}^{+}}=2 J_{\mathrm{H}_{2} \mathrm{O}_{4}}+J_{\mathrm{HCl}}+J_{\mathrm{HNO}_{3}}-J_{\mathrm{NH}_{3}}$

with $J_{i}$ the molar flux in species $\mathrm{X}_{\mathrm{i}}$. The $p H$ evolution due to c/e can be very stiff and cause instabilities, due to the very small quantity $n_{H^{+}}$of hydrogen ions inside the particle. The hydrogen ion flux is then limited to a given fraction $A$ of the hydrogen ion concentration following Pilinis et al. (2000) : $\left|J_{H^{+}}\right| \leq A n_{H^{+}}$, where $A$ is usually chosen arbitrarily between 0.01 and 0.1. $A$ is a numerical parameter that has no physical meaning and does not influence the final state of mass transfer. It just modifies the numerical path to reach this state. We refer to Pilinis et al. (2000) for a deeper understanding.

\subsubsection{Inorganic thermodynamics}

There are a range of packages available to solve thermodynamics for inorganic species Zhang et al. (1998). ISORROPIA Nenes et al. (1998) was shown to be a computationally efficient model that is also numerically accurate and stable and provides both a closed mode (for global equilibrium, a.k.a. forward mode) and open mode (for local equilibrium and kinetic mass transfer, a.k.a. reverse mode). Particles can be solid, liquid, both or in a metastable state, where particles are always in aqueous solution.

Moreover, the inclusion of sea salt $(\mathrm{NaCl})$ in the computation of thermodynamics is also an option in SIREAM.

When the particles are solid, fluxes of inorganic species are governed by gas/solid reactions at the particle surface. In this case, thermodynamic models are not able to compute gas equilibrium concentrations. For solid particle, SIREAM calculations are based on the solutions proposed in Pilinis et al. (2000).

\subsubsection{Secondary Organic Aerosols}

The oxidation of VOCs leads to species (SVOCs) that have increasingly complicated chemical functions, high polarizations, and lower saturation vapor pressure.

There are many uncertainties surrounding the formation of secondary organic aerosol. Due to the lack of knowledge and the sheer number and complexity of organic species, most chemical reaction schemes for organics are very crude representations of the "true" mechanism. These typically include the lumping of "representative" organic species and highly simplified reaction mechanisms.

The default gas-phase chemical mechanism for SIREAM is RACM Stockwell et al. (1997). Notice that the gas-phase mechanism and the related SVOCs are parameterized and can be easily modified.

The low volatility SOA precursors and the partitioning between the gas and particle phases are based on the empirical SORGAM model (Schell et al. (2001a); Schell (2000)). Eight SOA classes are taken into account (4 anthropogenic and 4 biogenic). Anthropogenic species include two from aromatic precursors (ARO 1 and ARO 2), one from higher alkanes (OLE 1) and one from higher alkenes (ALK 1). The biogenic species represent two classes from $\alpha$-pinene (API 1 and API 2) and two from limonene (LIM 1 and LIM 2) degradation. Some oxidation reactions of the form $\mathrm{VOC}+\mathrm{Ox} \rightarrow \mathrm{P}$ where $\mathrm{Ox}$ is $\mathrm{OH}, \mathrm{O}_{3}$, or NO3 have been modified to $\mathrm{VOC}+\mathrm{Ox} \rightarrow \mathrm{P}+\alpha_{1} \mathrm{P}_{1}+\alpha_{2} \mathrm{P}_{2}$ with $\mathrm{P} 1$ and $\mathrm{P} 2$ representing SVOCs among the eight classes. Updated values of these parameters have also been defined in other versions of the mechanism (not reported here).

The partitioning between the gas phase and the particle phase is performed in the following way. Let $n_{O M}$ be the number of organic species in the particle mixture (this in- 
cludes primary and secondary species) which are assumed to constitute an ideal mixture:

$\left(q_{i}\right)_{g}=\gamma_{i}\left(x_{i}\right)_{a} q_{i}^{\text {sat }}$

For species $\mathrm{X}_{\mathrm{i}}, q_{i}^{\text {sat }}$ is the saturation mass concentration in a pure mixture, $\left(x_{i}\right)_{a}$ is the molar fraction in the organic mixture and $\gamma_{i}$ is the activity coefficient in the organic mixture (a default value of 1 is assumed). $\left(x_{i}\right)_{a}$ is computed through:

$$
\left(x_{i}\right)_{a}=\frac{\frac{\left(q_{i}\right)_{a}}{M_{i}}}{\frac{q_{O M}}{M_{O M}}}=\frac{\frac{\left(q_{i}\right)_{a}}{M_{i}}}{\sum_{j=1}^{j=n_{O M}} \frac{\left(q_{j}\right)_{a}}{M_{j}}+\frac{\left(q_{P O A}\right)_{a}}{M_{P O A}}}
$$

$q_{O M}$ is the total concentration of organic matter (primary and secondary) in the particle phase. The molar mass $M_{i}$ of component $i$ is expressed in $\mu \mathrm{g} / \mathrm{mol}$ (in the same unit as the mass concentrations $q_{i}$ ); $M_{O M}$ is the average molar mass for organic matter in $\mu \mathrm{g} / \mathrm{mol}$. POA stands for the primary organic matter, assumed not to evaporate.

$q_{i}^{s a t}$ is computed from the saturation vapor pressure with $q_{i}^{s a t}=\frac{M_{i}}{R T} p_{i}^{\text {sat }}$. A similar way to proceed is to define the partitioning coefficient $K_{i}=\frac{\left(q_{i}\right)_{a}}{q_{O M}\left(q_{i}\right)_{g}}\left(\right.$ in $\left.^{3} / \mu \mathrm{g}\right)$. $K_{i}$ can be computed from the thermodynamic conditions and the saturation vapor pressure through:

$K_{i}=\frac{R T}{p_{i}^{s a t} \gamma_{i}\left(M_{O M}\right)}$

The saturation vapor pressure $p_{i}^{s a t}(T)$ is given by the Clausius-Clapeyron law:

$p_{i}^{\text {sat }}(T)=p_{i}^{\text {sat }}(298 K) \exp \left(-\frac{\Delta H_{\text {vap }}}{R}\left(\frac{1}{T}-\frac{1}{298}\right)\right)$

with $\Delta H_{v a p}$ the vaporization enthalpy (in the default version, a constant value $156 \mathrm{~kJ} / \mathrm{mol}$ ).

The mass concentration of a gas at local equilibrium with the particle mixture is given by Eq. (5). The global equilibrium between a gas and the particle mixture is given by Eq. (5) and mass conservation for species $X_{i}$ :

$\left(q_{i}\right)_{a}+\left(q_{i}\right)_{g}=\left(q_{i}\right)_{t o t}$

with $\left(q_{j}\right)_{t o t}$ representing the total mass concentration (for both phases) to be partitioned. This with Eq. (6) leads to a system of $n_{O M}$ algebraic equations of second degree:

$-a_{i}\left(\left(q_{i}\right)_{a}\right)^{2}+b_{i}\left(q_{i}\right)_{a}+c_{i}=0$

where the coefficients depend on concentrations $\left\{\left(q_{j}\right)_{a}\right\}_{j \neq i}$ through $a_{i}=\frac{1}{M_{i}}, \quad b_{i}=\frac{q_{i}^{\text {sat }}}{M_{i}}-\Sigma_{i}, \quad c_{i}=q_{i}^{\text {sat }} \Sigma_{i}$ and $\Sigma_{i}=\sum_{j=1, j \neq i}^{j=n_{O M}} \frac{\left(q_{j}\right)_{a}}{M_{j}}+\frac{\left(q_{P O A}\right)_{a}}{M_{P O A}}$.

The resulting system is solved by an iterative approach with a fixed point algorithm. Each second degree equation is solved in an exact way: the only positive root is computed for each equation of type (10).

\subsubsection{Wet diameter}

Parameterizations of coagulation, condensation/evaporation, dry deposition and wet scavenging depend on the particle "wet" diameter $d_{p}$. Two methods have been implemented in SIREAM to compute it, one based on thermodynamics, another on the Gerber's Formula.

The thermodynamic method consists in using the particle internal composition $\left\{m_{i}\right\}$ provided by the thermodynamic model ISORROPIA. Many of aerosol models use a constant specific particle mass $\rho_{p}$ Wexler et al. (1994); Pilinis and Seinfeld (1988) supposed to satisfy $\rho_{p} \frac{\pi d_{p}^{3}}{6}=\sum_{i=1}^{n_{e}} m_{i}$. In SIREAM, following Jacobson (2002), the particle volume is split into a solid part and a liquid part: $\frac{\pi d_{p}^{3}}{6}=V_{\text {liq }}+V_{\text {sol. }}$. As each solid represents one single phase, the total solid particle volume is the sum of each solid volume : $V_{\text {sol }}=\sum_{i_{s}} \frac{m_{i_{s}}}{\rho_{i_{s}}^{*}}$, with $\rho_{i_{s}}^{*}$ the specific mass of pure component $X_{i_{s}}$. The liquid particle phase is a concentrated mixing of inorganic species, whose volume is a non linear function of its inorganic internal composition : $V_{\text {liq }}=\sum_{i_{l}} V_{i_{l}} n_{i_{l}}$ where $V_{i_{l}}$ is the partial molar volume of ionic or dissolved species $X_{i_{l}}$ and $n_{i_{l}}$ is the molar quantity in $X_{i_{l}}$. Due to some molecular processes within the mixture (e.g. volume exclusion), the partial molar volume is a function of the internal composition. However, we assume that $V_{i_{l}} \simeq \frac{M_{i}}{\rho_{i_{l}}^{*}}$ where $M_{i}$ and $\rho_{i_{l}}^{*}$ are the molar mass of $X_{i}$ and the specific mass of a pure liquid solution of $X_{i}$, respectively. This method is well suited for condensation/evaporation for which thermodynamic computation cannot be avoided.

For other processes (coagulation, dry deposition and scavenging) the particle "wet" diameter is computed through a faster method, the Gerber's Formula (Gerber (1985)). This one is a parameterization of the "wet" radius as a function of the dry one :

$r_{w}=\left[\frac{C_{1}\left(r_{d}\right)^{C_{2}}}{C_{3}\left(r_{d}\right)^{C_{4}}-\log R H}+\left(r_{d}\right)^{3}\right]^{\frac{1}{3}}$

where $r_{w}$ and $r_{d}$ are respectively the wet and dry particle radius in centimeters, $R H$ is the atmospheric relative humidity within $[0,1]$. Coefficients $\left(C_{i}\right)_{i=1,4}$ depend on the particle type (urban, rural or marine). The $C_{3}$ coefficient is temperature dependent $(\mathrm{T})$ through the Kelvin effect:

$C_{3}(T)=C_{3}\left[1+C_{5}(298-T)\right]$

We have actually modified the coefficients given by Gerber through a minimization method so that the Gerber's Formula give results as close as possible to the "wet" diameters given by the thermodynamic method Sportisse et al. (2006):

$C_{1}=0.4989, C_{2}=3.0262, C_{3}=0.537210^{-12}$

$C_{4}=-1.3711, C_{5}=0.394210^{-02}$

The choice of which method to use (thermodynamics or Gerber's Formula) is up to the user. 


\subsubsection{Logarithmic formulation for the GDE}

On the basis of the parameterizations described above, the evolution of the number and mass distributions is governed by the GDE :

$$
\begin{aligned}
\frac{\partial n}{\partial t}(x, t)= & \int_{x_{0}}^{\tilde{x}} K(y, z) n(y, t) n(z, t) d y \\
& -n(x, t) \int_{x_{0}}^{\infty} K(x, y) n(y, t) d y \\
& -\frac{\partial\left(H_{0} n\right)}{\partial x} \\
\frac{\partial q_{i}}{\partial t}(x, t)= & \int_{x_{0}}^{\tilde{x}} K(y, z)\left[q_{i}(y, t) n(z, t)+n(y, t) q_{i}(z, t)\right] d y \\
& -q_{i}(x, t) \int_{x_{0}}^{\infty} K(x, y) n(y, t) d y \\
& -\frac{\partial\left(H_{0} q_{i}\right)}{\partial x}+\left(I_{i} n\right)(x, t)
\end{aligned}
$$

$H_{0}=\frac{I_{0}}{m}$ (in $\left.s^{-1}\right)$ is the logarithmic growth rate. The nucleation threshold is $x_{0}=\ln m_{0}$. Moreover, $\tilde{x}=\ln \left(e^{x}-e^{x_{0}}\right)$ and $z=\ln \left(e^{x}-e^{y}\right)$ in the above formula.

At the nucleation threshold, the nucleation rate determines the boundary condition :

$$
\left(H_{0} n\right)\left(x_{0}, t\right)=J_{0}(t), \quad\left(H_{0} q_{i}\right)\left(x_{0}, t\right)=m_{i}\left(x_{0}, t\right) J_{0}(t)
$$

The evolution of the gaseous concentration for the semivolatile species $\mathrm{X}_{\mathrm{i}}$ is given by:

$\frac{d c_{i}^{g}}{d t}(t)=-m_{i}\left(x_{0}, t\right) J_{0}(t)-\int_{x_{0}}^{\infty}\left(I_{i} n\right)(x, t) d x$

or by mass conservation : $c_{i}^{g}(t)+\int_{x_{0}}^{\infty} q_{i}(x, t) d x=K_{i}$.

\subsection{Other processes related to aerosols}

The following processes are not directly part of SIREAM. As such, the core of SIREAM (the parameterizations and the algorithms for the GDE) is independent. As for SOA, other parameterizations can be used. For the sake of completeness, we have chosen to include a brief description of the default current parameterizations.

2.3.1 Mass transfer and aqueous-phase chemistry for cloud droplets

For cells with a liquid water content exceeding a critical value (the default value is $0.05 \mathrm{~g} / \mathrm{m}^{3}$ ), the grid cell is assumed to contain a cloud and the aqueous-phase module is called instead of the SIREAM module. A part of the particle distribution is activated for particles that exceed a critical dry diameter the default value is $d_{\text {activ }}=0.7 \mu \mathrm{m}$ Strader et al. (1998). The microphysical processes that govern the evolution of cloud droplets are parameterized and not explicitly described. Cloud droplets form on activated particles and evaporate instantaneously (during one numerical timestep) in order to take into account the impact of aqueous-phase chemistry for the activated part of the particle distribution Fahey (2003); Fahey and Pandis (2001).

The activated particle fraction is then incorporated into the cloud droplet distribution. The VSRM model can simulate a size-resolved droplet distribution, but we use only a bulk approach in order to decrease the computational. In this case the average droplet diameter is fixed at $20 \mu \mathrm{m}$. The chemical composition of the cloud droplet is then given by the activated particle fraction.

Aqueous-phase chemistry and mass transfer between the gaseous phase and the cloud droplets (bulk solution) are then solved. The aqueous-phase model is based on the chemical mechanism developed at Carnegie Mellon University Strader et al. (1998). It contains 18 gas-phase species and 28 aqueous-phase species. Aqueous-phase chemistry is modeled by a chemical mechanism of 99 chemical reactions and 17 equilibria (for ionic dissociation).

Mass transfer is solved dynamically only for "slow"1 species, while "fast" species are assumed to be described by Henry's equilibrium, we refer to Sportisse et al. (2006) for a detailed list of species and their status.

The radical chemistry is not taken into account. The computation of $\mathrm{H}+$ is made with the electroneutrality relation written as $f_{\text {electroneutrality }}\left(H^{+}\right)=0$. This nonlinear algebraic equation is solved with the bisection method. If no convergence occurs, we take a default value $p H=4.5$ as observed droplet $\mathrm{pH}$ often ranges between 4.0 en 5.0 Seinfeld and Pandis (1998); Pruppacher and Klett (1998).

After one timestep, the new mass generated from aqueous chemistry is redistributed onto aerosol bins that were activated. To do so the initial aerosol distribution is assumed to have a bimodal shape (log-normal distributions) that gives weighting factors for each aerosol bin. Median diameter and variance for each mode are respectively $0.4 \mu \mathrm{m}$ and 1.8 for first one, $2.5 \mu \mathrm{m}$ and 2.15 for second one. The tests in Fahey (2003) illustrate the low impact of the choice made for this assumption.

We use a splitting method, the gas-phase chemistry being solved elsewhere (in the gas-phase module of the ChemistryTransport Model). Aqueous-phase chemistry and mass transfer are solved with VODE Brown et al. (1989).

\subsubsection{Heterogeneous reactions}

The heterogeneous reactions at the surface of condensed matter (particles and cloud or fog droplets) may significantly impact gas-phase photochemistry and particles. This process is solved together with gas-phase chemistry. Following Jacob

\footnotetext{
1 "slow" and "fast" refer to the time for given species to reach equilibrium
} 
(2000), these processes are described by the first-order reactions:

$$
\begin{array}{rll}
\mathrm{HO}_{2} & \stackrel{\mathrm{PM}}{\rightarrow} & 0.5 \mathrm{H}_{2} \mathrm{O}_{2} \\
\mathrm{NO}_{2} & \stackrel{\mathrm{PM}}{\rightarrow} & 0.5 \mathrm{HONO}+0.5 \mathrm{HNO}_{3} \\
\mathrm{NO}_{3} & \stackrel{\mathrm{PM}}{\rightarrow} & \mathrm{HNO}_{3} \\
\mathrm{~N}_{2} \mathrm{O}_{5} & \stackrel{\mathrm{PM}, \text { clouds }}{\longrightarrow} & 2 \mathrm{HNO}_{3}
\end{array}
$$

The heterogeneous reactions for $\mathrm{HO} 2, \mathrm{NO} 2$ and $\mathrm{NO} 3$ at the surface of cloud droplets are assumed to be taken into account in the aqueous-phase model and are considered separately.

The first-order kinetic rate is computed for gas-phase species $\mathrm{X}_{\mathrm{i}}$ with $k_{i}=\left(\frac{a}{D_{i}^{g}}+\frac{4}{\bar{c}_{i}^{g} \gamma}\right)^{-1} S_{a}$ where $a$ is the particle radius, $\bar{c}_{i}^{g}$ the thermal velocity in the air, $\gamma$ the reaction probability and $S_{a}$ the available surface for condensed matter per air volume.

$\gamma$ strongly depends on the chemical composition and on the particle size. We have decided to keep the variation ranges from Jacob (2000) for these parameters in order to evaluate the resulting uncertainties: $\gamma_{\mathrm{HO}} \in[0.1-$ $1], \gamma_{N O 2} \in\left[10^{-6}-10^{-3}\right], \gamma_{N O 3} \in\left[2.10^{-4}-10^{-2}\right]$ and $\gamma_{N 2 O 5} \in[0.01-1]$. The default values are the lowest values. For numerical stability requirements, these reactions are coupled to the gas-phase mechanism.

\section{Numerical simulation}

\subsection{Numerical strategy}

On the basis of a comprehensive benchmark of algorithms Debry (2004), the numerical strategy relies on methods that ensure stability with a low CPU cost. First, we use a splitting approach for coagulation and condensation/evaporation. Second, the discretization is performed with sectional methods which remain stable even with a few discretization points, contrary to spectral methods Sandu and Borden (2003); Debry and Sportisse (2005b). Third, condensation/evaporation is solved with a Lagrangian method, the quasistationary method of Jacobson Jacobson (1997) is employed to reduce the numerical diffusion associated with $\mathrm{Eu}-$ lerian schemes in the case of a small number of discretization points (typically the case in 3D models).

The splitting sequence goes from the slowest process to the fastest one (first coagulation and then condensation/evaporation-nucleation). The nucleation process is not a numerical issue and is solved simultaneously with condensation/evaporation. In the following, we present the numerical algorithm used for each process.

The particle mass distribution is discretized into $n_{b}$ bins $\left[x^{j}, x^{j+1}\right]$. We define the integrated quantities over the bin $j$ for the number distribution and the mass distributions for species $X_{i}$ :

$N^{j}(t)=\int_{x^{j}}^{x^{j+1}} n(x, t) d x, \quad Q_{i}^{j}=\int_{x^{j}}^{x^{j+1}} q_{i}(x, t) d x$

$\tilde{m}_{i}^{j}=\frac{Q_{i}^{j}}{N^{j}}$ is the average mass per particle inside bin $j$ for species $X_{i}$.

We use a Method of Lines by first performing size discretization and then time integration. After discretization, the resulting system of Ordinary Differential Equations (ODEs) has the generic form:

$\frac{d c}{d t}=f(c, t)$

where the state vector $c$ is specific for each process. $c_{n}$ is the numerical approximation of $c\left(t_{n}\right)$ at time $t_{n}$, with a timestep $\Delta t_{n}=t_{n+1}-t_{n}$. A second-order solver is specified for each case with a first-order approximation $\tilde{c}_{n+1}$. The variable timestep $\Delta t_{n}$ is adjusted by:

$\Delta t_{n+1}=\Delta t_{n} \sqrt{\frac{\varepsilon_{r}\left\|c_{n+1}\right\|_{2}}{\left\|\tilde{c}_{n+1}-c_{n+1}\right\|_{2}}}$

where $\varepsilon_{r}$ is a user parameter, usually between 0.01 and 0.5 . The higher $\varepsilon_{r}$ is, the faster $\Delta t_{n}$ increases. \|\|$_{2}$ is the Euclidean norm.

\subsection{Size discretization}

\subsubsection{Coagulation}

Coagulation is solved by the so-called size binning method (Jacobson et al. (1994)). Equations (14) and (15) are integrated over each bin, which gives:

$$
\begin{aligned}
& \frac{d N^{k}}{d t}(t)=\frac{1}{2} \sum_{j_{1}=1}^{k} \sum_{j_{2}=1}^{k} f_{j_{1} j_{2}}^{k} K_{j_{1} j_{2}} N^{j_{1}} N^{j_{2}}-N^{k} \sum_{j=1}^{n_{b}} K_{k j} N^{j} \\
& \frac{d Q_{i}^{k}}{d t}(t)=\sum_{j_{1}=1}^{k} \sum_{j_{2}=1}^{k} f_{j_{1} j_{2}}^{k} K_{j_{1} j_{2}} Q_{i}^{j_{1}} N^{j_{2}}-Q_{i}^{k} \sum_{j=1}^{n_{b}} K_{k j} N^{j}(21)
\end{aligned}
$$

$K_{j_{1} j_{2}}$ is an approximation of the coagulation kernel between bins $j_{1}$ and $j_{2}$.

The key point is to compute the partition coefficients $f_{j_{1} j_{2}}^{k}$ that represent the fraction of particle combinations between bins $j_{1}$ and $j_{2}$ falling into bin $k$. As these coefficients only depend on the chosen discretization, they can be computed in a preprocessed step. The computation depends on the assumed shape of continuous densities inside each bin for the closure scheme, see Debry and Sportisse (2005a). In SIREAM, we use a closure scheme similar to Fernndez-Daz et al. (2000). 


\subsubsection{Condensation/evaporation-nucleation}

Lagrangian formulation Let $\bar{x}^{j}(t)$ be the logarithmic mass of one particle at time $t$ whose initial value corresponds to point $x^{j}$ of the fixed discretization. The time evolution of $\bar{x}^{j}(t)$ is given by the equation of the characteristic curve :

$\frac{d \bar{x}^{j}}{d t}(t)=H_{0}\left(\bar{x}^{j}, t\right), \bar{x}^{j}(0)=x^{j}$

One crucial issue is to ensure that the characteristic curves do not cross themselves. If this happens the Lagrangian formulation is no longer valid. In real cases we have no proof that this does not happen, even though we have not seen such a situation up to now.

Provided that the characteristic curves do not cross, we can define integrated quantities $N^{j}$ and $Q_{i}^{j}$ for each La-

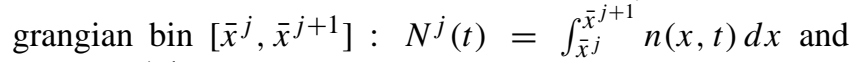
$Q_{i}^{j}=\int_{\bar{x}^{j}}^{\bar{x}^{j+1}} q_{i}(x, t) d x$.

Mass conservation can be easily written in the form : $c_{i}^{g}(t)+\sum_{j=1}^{n_{b}} Q_{i}^{j}(t)=K_{i}$.

The time derivation of integrated quantities leads to the equations:

$$
\frac{d N^{j}}{d t}=0, \frac{d Q_{i}^{j}}{d t}=N^{j} \tilde{I}_{i}^{j}
$$

$\tilde{I}_{i}^{j}$ is an approximation of the mass transfer rate for species $X_{i}$ in bin $j$ :

$$
\tilde{I}_{i}^{j}=\underbrace{2 \pi D_{i} d_{p}^{j} f\left(K_{n_{i}}^{j}, \alpha_{i}\right)}_{a_{i}^{j}}\left(K_{i}-\sum_{k=1}^{n_{b}} Q_{i}^{k}-\eta^{j}\left(c_{i}^{e q}\right)^{j}\right)
$$

with $\eta^{j}=e^{\frac{4 \sigma v_{p}}{R T d_{p}^{j}}} \cdot\left(c_{i}^{e q}\right)^{j}$ is computed at $\tilde{m}_{i}^{j}$.

For the nucleation process, the first bound $x^{1}$ is assumed to correspond to the nucleation threshold, so that the Lagrangian bound $\bar{x}^{1}$ does not satisfy (22) but:

$\frac{d \bar{x}^{1}}{d t}=j(t), \quad \bar{x}^{1}(0)=x^{1}$

where $j(t)$ is the growth law of the first bound due to nucleation and given by the nucleation parameterization. The equations for the first Lagrangian bin therefore are written as:

$\frac{d N^{1}}{d t}=J_{0}(t), \quad \frac{d Q_{i}^{1}}{d t}=N^{1} \tilde{I}_{i}^{1}+m_{i}\left(x^{1}, t\right) J_{0}(t)$

where $\left[m_{1}\left(x^{1}, t\right), \ldots, m_{n_{e}}\left(x^{1}, t\right)\right]$ is the chemical composition of the nucleated particles, also given by the nucleation process.

The Lagrangian formulation consists in solving Eqs. (22), (23) and (26). In the next section we detail the various numerical strategies to perform the time integration, which is by far the most challenging point in particle simulation.
Interpolation of Lagrangian boundaries One has to solve the equations for the characteristic curves in order to know the boundaries of each bin. Notice that the c/e equations for boundaries are similar to those for integrated quantities. Indeed, for $j=1, \ldots, n_{b}$ and $\tilde{x}^{j}=\ln \left(\tilde{m}^{j}\right)$, one gets from Eq. (23):

$$
\frac{d \tilde{x}^{j}}{d t}=\tilde{H}_{0}^{j}, \quad \tilde{H}_{0}^{j}=\frac{\tilde{I}_{0}^{j}}{\tilde{m}^{j}}
$$

In practice, in order to reduce the computational burden, one tries to avoid solving boundary equations. An alternative is to interpolate the bin boundaries from integrated quantities.

First method Koo et al. (2003) consists of utilizing the geometric mean of two adjacent bin :

for $j=2, \ldots, n_{b}, \quad \bar{m}^{j}(t)=\sqrt{\tilde{m}^{j-1}(t) \tilde{m}^{j}(t)}$

This algorithm would have a physical meaning if Eqs. (22) and (27) were conserving formula (28), which is not the case. We have therefore developed another algorithm.

Equations (22) and (27) are similar and therefore $\tilde{x}^{j}$ and $\bar{x}^{j}$ evolve in the same proportion given by $\lambda^{j}(t)(j \geq 2)$ :

$\lambda^{j}(t)=\frac{\bar{x}^{j}(t)-\tilde{x}^{j-1}(t)}{\tilde{x}^{j}(t)-\tilde{x}^{j-1}(t)}$

$\lambda^{j}(0)$ is known because $\bar{x}^{j}(0)=x^{j}$. The time integration over $[0, t]$ of Eqs. (22) and (27) gives for $j \geq 1$ :

$\bar{x}^{j}(t)=x^{j}+\Delta \bar{x}^{j}, \Delta \bar{x}^{j}=\int_{0}^{t} H_{0}^{j}\left(t^{\prime}\right) d t^{\prime}$
$\tilde{x}^{j}(t)=\tilde{x}^{j}(0)+\Delta \tilde{x}^{j}, \Delta \tilde{x}^{j}=\int_{0}^{t} \tilde{H}_{0}^{j}\left(t^{\prime}\right) d t^{\prime}$

The variation of each boundary $\bar{x}^{j}$ is then computed from that of its two adjacent bins $\tilde{x}^{j-1}$ and $\tilde{x}^{j}$ :

$\Delta \bar{x}^{j} \simeq\left(1-\lambda^{j}(0)\right) \Delta \tilde{x}^{j-1}+\lambda^{j}(0) \Delta \tilde{x}^{j}$

where one assumes that $\lambda^{j}$ remains constant.

Redistribution on a fixed size grid Using a Lagrangian approach for condensation/evaporation requires the redistribution or projection of number and mass concentrations onto the fixed size grid required by a 3D model or for coagulation.

Let $N$ and $\left(Q_{i}\right)_{i=1}^{n_{e}}$ be the integrated quantities of one Lagrangian bin after condensation/evaporation. We assume that this Lagrangian bin is covered by two adjacent fixed bins labelled $j$ and $j+1$.

The redistribution algorithm must be conservative for the number and mass distribution of species $X_{i}$ :

$N=N^{j}+N^{j+1}, \quad Q_{i}=Q_{i}^{j}+Q_{i}^{j+1}$

Two algorithms have been developed: 
1. If $\bar{x}_{l o}$ and $\bar{x}_{h i}$ are the boundaries of the Lagrangian bin after condensation/evaporation, the redistribution is performed as follows for the number distribution and the mass distribution of species $X_{i}$ :

$$
\begin{aligned}
N^{j} & =\frac{\bar{x}_{h i}^{j}-\bar{x}_{l o}}{\bar{x}_{h i}-\bar{x}_{l o}} N, \quad Q_{i}^{j}=\frac{\bar{x}_{h i}^{j}-\bar{x}_{l o}}{\bar{x}_{h i}-\bar{x}_{l o}} Q_{i} \\
N^{j+1} & =\frac{\bar{x}_{h i}-\bar{x}_{l o}^{j+1}}{\bar{x}_{h i}-\bar{x}_{l o}} N, Q_{i}^{j+1}=\frac{\bar{x}_{h i}-\bar{x}_{l o}^{j+1}}{\bar{x}_{h i}-\bar{x}_{l o}} Q_{i}
\end{aligned}
$$

The number and aerosol mass are redistributed in equal proportions, depending upon the part of each fixed bins covered by the Lagrangian one. Nevertheless this implies that the average masses of fixed bins be equal to the Lagrangian bin one $(Q / N)$ and may fall out of fixed bin boundaries.

2. Another approach consists in conserving the average mass. Let $\tilde{m}=Q / N$ be the averaged Lagrangian bin, $\tilde{m}^{j}$ and $\tilde{m}^{j+1}$ be respectively the centered mass of bins $j$ and $j+1$. The number distribution and the mass distribution of species $X_{i}$ is redistributed ensuring conservation of relations $Q^{j}=\tilde{m}^{j} N^{j}$ and $Q^{j+1}=\tilde{m}^{j+1} N^{j+1}$, which together with (32) lead to the algorithm:

$$
\begin{gathered}
N^{j}=\frac{1-\frac{\tilde{m}}{\tilde{m}^{j+1}}}{1-\frac{\tilde{m}^{j}}{\tilde{m}^{j+1}}} N, Q_{i}^{j}=\frac{\frac{\tilde{m}^{j+1}}{\tilde{m}}-1}{\frac{\tilde{m}^{j+1}}{\tilde{m}^{j}}-1} Q_{i} \\
N^{j+1}=\frac{1-\frac{\tilde{m}}{\tilde{m}^{j}}}{1-\frac{\tilde{m}^{j+1}}{\tilde{m}^{j}}} N, Q_{i}^{j+1}=\frac{1-\frac{\tilde{m}^{j}}{\tilde{m}}}{1-\frac{\tilde{m}^{j}}{\tilde{m}^{j+1}}} Q_{i}
\end{gathered}
$$

This algorithm comes to the fitting method developed by Jacobson in Jacobson (1997).

The first method takes advantage of the more sophisticated computation of bound sections but does not conserve average mass. The second method conserves average mass but may increase numerical diffusivity due to the lack of bound section information.

Both schemes are available in SIREAM.

\subsection{Time integration}

\subsubsection{Coagulation}

As coagulation is not a stiff process, we solve it by the second order explicit scheme ETR (Explicit Trapezoidal Rule) with the sequence:

$\tilde{c}_{n+1}=c_{n}+\Delta t f\left(c_{n}, t_{n}\right)$

$c_{n+1}=c_{n}+\frac{\Delta t}{2}\left(f\left(c_{n}, t_{n}\right)+f\left(\tilde{c}_{n+1}, t_{n+1}\right)\right)$

with $c=\left(N^{1}, \ldots, N^{n_{b}}, Q_{1}^{1}, \ldots, Q_{1}^{n_{b}}, \ldots, Q_{n_{e}}^{1}, \ldots, Q_{n_{e}}^{n_{b}}\right)$.

\subsubsection{Condensation/evaporation}

Here, $c=\left(Q_{1}^{1}, \ldots, Q_{n_{e}}^{1}, \ldots, Q_{1}^{n_{b}}, \ldots, Q_{n_{e}}^{n_{b}}\right)^{T} . n_{c}=n_{e} \times$ $n_{b}$ is the dimension of $c$.

SIREAM offers three methods for solving condensation/evaporation: a fully dynamic method that treats dynamic mass transfer for each bin, a bulk equilibrium approach, and a hybrid approach that combines the two previous approaches.

Fully dynamic method Due to the wide range of timescales related to mass transfer, the system is stiff and implicit algorithms have to be used. The second-order Rosenbrock scheme Verwer et al. (1999); Djouad et al. (2002), ROS2, is applied for the time integration :

$$
\begin{aligned}
c_{n+1}= & c_{n}+\frac{\Delta t_{n}}{2}\left(3 k_{1}+k_{2}\right) \\
& {\left[I-\gamma \Delta t_{n} J(f)\right] k_{1}=f\left(c_{n}, t_{n}\right) } \\
& {\left[I-\gamma \Delta t_{n} J(f)\right] k_{2}=f\left(\tilde{c}_{n+1}, t_{n+1}\right)-2 k_{1} }
\end{aligned}
$$

where $\tilde{c}_{n+1}=c_{n}+\Delta t_{n} k_{1}$ and $\gamma=1+\frac{1}{\sqrt{2}}$.

This scheme requires the computation of the Jacobian matrix of $f$ (a matrix $n_{c} \times n_{c}$ ) defined by $[J(f)]_{k l}=\frac{\partial f^{k}}{\partial c^{l}} . f^{k}$ is the $k$ th component of function $f$ and $c^{l}$ is the $l$ th component of $c$.

Let us write $k=(i-1) n_{b}+j$ and $l=\left(i^{\prime}-1\right) n_{b}+j^{\prime}$ where $i$ and $i^{\prime}$ label the semi-volatile species while $j$ and $j^{\prime}$ label the bins. The $(k l)$ th element of the Jacobian matrix may then be written as

$\frac{\partial f^{k}}{\partial c^{l}}=\frac{\partial I_{i}^{j}}{\partial Q_{i^{\prime}}^{j^{\prime}}}$

The derivation of $f^{k}$ may be split into one linear part, due to mass conservation, and one non-linear part related to the coefficient $a_{i}^{j}$, to the Kelvin effect $\eta^{j}$, and to the gas equilibrium concentration $\left(c_{i}^{e q}\right)^{j}$. The linear part is analytically derived :

$\left(\frac{\partial f^{k}}{\partial c^{l}}\right)_{\operatorname{lin}}=-a_{i}^{j} N^{j^{\prime}}$

The non-linear part has to be differentiated by numerical methods, like the finite difference method :

$\left(\frac{\partial f^{k}}{\partial c^{l}}\right)_{\text {non-lin }}=\frac{f^{k}\left(\ldots, c^{l}\left(1+\varepsilon_{\mathrm{jac}}\right), \ldots\right)-f^{k}\left(\ldots, c^{l}, \ldots\right)}{c^{l} \varepsilon_{\mathrm{jac}}}$

where $\varepsilon_{\mathrm{jac}}$ is generally close to $10^{-8}$. During the numerical computation, the linear part is arbitrarily kept constant to avoid deriving it twice.

A default option, advocated for 3D applications, is to approximate the Jacobian matrix by its diagonal. The motivation here is to reduce the CPU time. 
Hybrid resolution Solving the c/e system, even with an implicit scheme, can be computationally inefficient. In order to lower the stiffness, hybrid methods for condensation/evaporation have been developed Capaldo et al. (2000). The method consists in partitioning the state vector $c$ into its fast components $\left(c^{f}\right)$ and its slow components $\left(c^{s}\right)$ respectively:

$$
\frac{d c^{s}}{d t}=f^{s}\left(c^{s}, c^{f}, t\right), \quad f^{f}\left(c^{s}, c^{f}, t\right)=0
$$

The algebraic equation states that the fast part is a function of the slow part, $c^{f}(t)=g\left(c^{s}(t), t\right)$. The time evolution of the slow part is now governed by :

$$
\frac{d c^{s}}{d t}=f^{s}\left(c^{s}, g\left(c^{s}(t), t\right), t\right)
$$

As $c^{s}$ gathers particle species and sizes which have a slow c/e characteristic time, stiffness is substantially reduced.

The issue is now to determine whether particle sizes and species are "slow" or "fast". The spectral study of the c/e system Debry and Sportisse (2006) indicates how to compute a cutting diameter $d_{c}$ between "slow" and "fast" species/sizes, such that the partitioning consists of cutting the particle distribution as follows: the smallest bins are at equilibrium while the coarsest ones are governed by kinetic mass transfer. The cutting diameter can be computed by QSSA criteria, defined by :

$$
\operatorname{QSSA}_{i}^{j}=\frac{c_{i}^{g}-\eta_{i}^{j}\left(c_{i}^{e q}\right)^{j}}{c_{i}^{g}+\eta_{i}^{j}\left(c_{i}^{e q}\right)^{j}}
$$

for a given chemical species $X_{i}$ and one particle size $j$. The closer this ratio to unity, the closer the species and the size are to equilibrium.

In practice all bins $j$ for which $\left(Q S S A_{i}^{j}\right)_{i=1}^{n_{e}}$ is greater than one, the user parameter $\varepsilon_{Q S S A}$ (close to unity) will be considered fast and solved by an equilibrium equation. In the following we write $j_{c}$ as the bin corresponding to the cutting diameter. Bin $j_{c}$ is the largest fast bin and bin $j_{c}+1$ is the smallest slow bin.

In SIREAM (to be used in 3D modeling), the default option is a fixed cutting diameter ( 1.25 or $2.5 \mu \mathrm{m})$.

The thermodynamic equilibrium between the gas phase and the fast particle bins is now written for species $X_{i}$ as:

$$
K_{i}^{f}-\sum_{j=1}^{j_{c}} Q_{i}^{j}-\eta_{i}^{k} c_{i}^{e q}\left(Q_{1}^{k}, \ldots, Q_{n_{e}}^{k}\right)=0
$$

with $K_{i}^{f}=K_{i}-\sum_{j=j_{c}+1}^{n_{b}} Q_{i}^{j}$ the total mass of species $X_{i}$ for fast bins.

There are two approaches for solving this equilibrium: the bulk equilibrium approach and the size-resolved particle approach. For the size-resolved approach, we refer to Jacobson et al. (1996) (with the use of the fixed point algorithm) and to Debry and Sportisse (2006) (with a minimization procedure).

In SIREAM, the bulk equilibrium has been implemented (Pandis et al. (1993)). It consists in merging all fast bins $j \leq j_{c}$ into one bin, referred as the "bulk" aerosol phase :

$$
1 \leq i \leq n_{e}, \quad B_{i}=\sum_{j=1}^{j_{c}} Q_{i}^{j}
$$

The thermodynamic model ISORROPIA is then applied to the "bulk" aerosol phase $\left(B_{i}\right)_{i=1}^{n_{e}}$ and one gets equilibrium "bulk" concentrations $\left(B_{i}^{e q}\right)_{i=1}^{n_{e}}$ with the forward mode of the thermodynamics solver (global equilibrium).

The variation from initial to final "bulk" concentrations is then redistributed among fast bins $1 \leq k \leq j_{c}$ for species $X_{i}$ Pandis et al. (1993):

$$
\left(Q_{i}^{k}\right)^{e q}=Q_{i}^{k}+b_{i}^{k}\left(B_{i}^{e q}-B_{i}\right), b_{i}^{k}=\frac{a_{i}^{k} N^{k}}{\sum_{j=1}^{j_{c}} a_{i}^{j} N^{j}}
$$

This redistribution scheme is exact provided that the particle composition is uniform over fast bins and that the variation of the particle diameter can be neglected for fast bins Debry and Sportisse (2006).

Bulk approach It is a special case of the hybrid approach with the cutting diameter $j_{c}=1$ (all bins are at equilibrium).

\section{Implementation}

The SIREAM module is written entirely in fortran 77 . Its external dependencies are the thermodynamic module ISORROPIA (version 1.7 currently used) Nenes et al. (1998) and the VODE solver from the ODEPACK ordinary differential equation package ("double precision" version required). ISORROPIA is not distributed with SIREAM and has to be retrieved by the user on the ISORROPIA website (http://nenes.eas.gatech.edu/ISORROPIA/). The VODE solver can be retrieved from http://www.llnl.gov/CASC/ software.html, but as this solver is in the public domain we also freely ship it together with SIREAM.

\section{Conclusions}

We have summarized the main features of the aerosol model SIREAM (SIze REsolved Aerosol Model). SIREAM simulates the GDE for atmospheric particles and can be easily linked to a three-dimensional Chemistry-Transport-Model. Moreover, the physical parameterizations used by SIREAM can be easily modified. They are currently hosted by the library ATMODATA and shared by another aerosol model MAM, Sartelet et al. (2006).

The next development steps are related to the improvement of the modeling of Secondary Organic Aerosol. The current parameterization of SOA is limited because it does not 
take into account the hydrophilic behavior of organic species Griffin et al. (2002b,a); Pun et al. (2002). Furthermore new gas precursors such as isoprene and sesquiterpene should be added.

The modularity of SIREAM will be also strengthened by adding new alternative parameterizations (such as other thermodynamics models or simplified aqueous-phase chemical mechanisms) and new numerical algorithms (especially for time integration of condensation/evaporation).

A further step is also the extension to externally mixed aerosol.

Acknowledgements. Part of this project has been funded by the French Research Program, Primequal-Predit, in the framework of the PAM Project (Multiphase Air Pollution). Some of the authors (K. Fahey, K. Sartelet and M. Tombette) have been partially funded by the Region Ile de France.

Edited by: R. MacKenzie

\section{References}

Adams, P. and Seinfeld, J.: Predicting global aerosol size distributions in general circulation models, J. Geophys. Res., 107, 4370, 2002.

Binkowski, F. and Roselle, S.: Models-3 Community Multiscale Air Quality (CMAQ) model aerosol component. 1. Model description, J. Geophys. Res., 108, doi:10.1029/2001JD001 409, 2003.

Brown, P., Byrne, G., and Hindmarsh, A.: VODE: A Variable Coefficient ODE Solver, SIAM J. on Sci. and Stat. Comp., 10, 10381051, 1989.

Byun, P. and Schere, K.: EPA's Third Generation Air Quality Modeling System: Description of the Models-3 Community, J. Mech. Review, 2004.

Capaldo, K., Pilinis, C., and Pandis, S.: A computationally efficient hybrid approach for dynamic gas/aerosol transfer in air quality models, Atmos. Environ., 34, 3617-3627, 2000.

Dahneke, B.: Theory of Dispersed Multiphase Flow, Academic press, New York, 1983.

Debry, E.: Numerical simulation of an atmospheric aerosol distribution, Ph.D. thesis, ENPC, CEREA, in French, 2004.

Debry, E. and Sportisse, B.: Solving aerosol coagulation with sizebinning methods, Appl. Numer. Math., accepted, 2005a.

Debry, E. and Sportisse, B.: Numerical simulation of the General Dynamics Equation (GDE) for aerosols with two collocation methods, Appl. Numer. Math., accepted, 2005b.

Debry, E. and Sportisse, B.: Reduction of the condensation/evaporation dynamics for atmospheric aerosols: theoretical and numerical investigation of hybrid methods, J. Aerosol Sci., 37, 950-966, 2006.

Djouad, R., Sportisse, B., and Audiffren, N.: Numerical simulation of aqueous-phase atmospheric models : use of a non-autonomous Rosenbrock method, Atmos.Environ., 36, 873-879, 2002.

Fahey, K.: Cloud and fog processing of aerosols: modeling the evolution of atmospheric species in the aqueous phase, $\mathrm{Ph} . \mathrm{D}$. thesis, Carnegie Mellon University, 2003.
Fahey, K. and Pandis, S.: Optimizing model performance: variable size resolution in cloud chemistry modeling, Atmos. Environ., 35, 4471-4478, 2001.

Fernndez-Daz, J., Gonzlez-Pola Muiz, C., Rodrguez Braa, M., Arganza Garca, B., and Garca Nieto, P.: A modified semi-implicit method to obtain the evolution of an aerosol by coagulation, Atmos. Environ., 34, 4301-4314, 2000.

Gaydos, T., Koo, B., Pandis, S., and Chock, D.: Development and application of an efficient moving sectional approach for the solution of the atmospheric aerosol condensation/evaporation equations., Atmos. Environ., 37, 3303-3316, 2003.

Gelbard, F., Tambour, Y., and Seinfeld, J.: Sectional Representations for Simulating Aerosol Dynamics, Journal of collod and Interface Science, 76, 541-556, 1980.

Gerber, H.: Relative-humidity parameterization of the Navy aerosol model (NAM), Tech. Rep. 8956, Natl. Res. Lab. Washington D.C., 1985.

Gong, S., Barrie, L.A., and Blanchet, J.-P., et al.: Canadian Aerosol Module: a size-segregated simulation of atmospheric aerosol processes for climate and air quality models. I Module development, J. Geophys. Res., 108, 2003.

Griffin, R., Dabdub, D., Kleeman, M., Fraser, M., Cass, G., and Seinfeld, J.: Secondary organic aerosol 3. Urban/regional scale model of size- and composition-resolved aerosols, J. Geophys. Res., 107, 2002a.

Griffin, R., Dabdub, D., and Seinfeld, J.: Secondary organic aerosol 1. Atmospheric chemical mechanism for production of molecular constituents, J. Geophys. Res., 107, 2002b.

Jacob, D.: Heterogeneous chemistry and tropospheric ozone, Atmos. Environ, 34, 2131-2159, 2000.

Jacobson, M.: Development and application of a new air pollution modeling system - PART II . aerosol module structure and design, Atmospheric Environment, 31, 131-144, 1997.

Jacobson, M.: Analysis of aerosol interactions with numerical techniques for solving coagulation, nucleation, condensation, dissolution, and reversible chemistry among multiple size distributions, J. Geophys. Res., 107, 2002.

Jacobson, M., Turco, R., Jensen, E., and Toon, O.: Modeling coagulation among particles of different composition and size, Atmos. Environ., 28, 1327-1338, 1994.

Jacobson, M., Tabazadeh, A., and Turco, R.: Simulating equilibrium within aerosols and nonequilibrium between gases and aerosols, J. Geophys. Res., 101, 9079-9091, American Geophysical Union, 1996.

Koo, B., Gaydos, T., and Pandis, S.: Evaluation of the Equilibrium, Dynamic, and Hybrid aerosol modeling approaches, Aerosol Science Technology, 37, 53-64, 2003.

Korhonen, H., Lehtinen, K., Pirjola, L., Napari, I., Vehkamaki, H., Noppel, M., and Kulmala, M.: Simulation of atmospheric nucleation mode: a comparison of nucleation models and size distribution representations, J. Geophys. Res., 108, doi:10.1029/2002JD003 305, 2003.

Mallet, V. and Sportisse, B.: Data processing and parameterizations in atmospheric chemistry and physics: the AtmoData library, Tech. Rep. 2005-12, ENPC/CEREA, 2005.

Mallet, V. and Sportisse, B.: Toward ensemble-based air-quality forecasts, J. Geophys. Res., accepted, 2006.

Mallet, V., Quélo, D., Sportisse, B., Debry, É., Korsakissok, I., Wu, L., Roustan, Y., Sartelet, K., Tombette, M., de Biasi, M. A., and 
Foudhil, H.: Technical note: The air quality modeling system Polyphemus, Tech. Rep. 8, CEREA, 2007.

Meng, Z., Dabdub, D., and Seinfeld, J.: Size-resolved and chemically resolved model of atmospheric aerosol dynamics, J. Geophys. Res., 103, 3419-3435, 1998.

Napari, I., Noppel, M., Vehkamki, H., and Kulmala, M.: Parametrization of ternary nucleation rates for $\mathrm{H}_{2} \mathrm{O}_{4}-\mathrm{NH}_{3}-\mathrm{H}_{2} \mathrm{O}$ vapors, J. Geophys. Res., 107, 2002.

Nenes, A., Pandis, S., and Pilinis, C.: ISORROPIA : A new Thermodynamic Equilibrium Model for Multicomponent Inorganic Aerosols, Aquatic geochemistry, 4, 123-152, 1998.

Pandis, S., Wexler, A., and Seinfeld, J.: Secondary organic aerosol formation and transport -II. Predicting the ambient secondary organic aerosol size distribution, Atmos. Environ., 27A, 24032416, 1993.

Pilinis, C. and Seinfeld, J.: Development and evaluation of an eulerian photochemical gas-aerosol model, Atmos. Environ., 22, 1985-2001, 1988.

Pilinis, C., Capaldo, K., Nenes, A., and Pandis, S.: MADM - a new multi-component Aerosol Dynamic Model, Aerosol Science and Technology, 32, 482-502, 2000.

Pruppacher, H. and Klett, J.: Microphysics of Clouds and Precipitation, Kluwer Academic Publishers, 1998.

Pun, B., Griffin, R., Seigneur, C., and Seinfeld, J.: Secondary organic aerosol 2. Thermodynamic model for gas/particle partitioning of molecular constituents, J. Geophys. Res., 107, 2002.

Sandu, A. and Borden, C.: A framework for the numerical treatment of aerosol dynamics, Appl. Numer. Math., 45, 475-497, 2003.

Sartelet, K. N., Hayami, H., Albriet, B., and Sportisse, B.: Development and preliminary validation of a Modal Aerosol Model for tropospheric chemistry: MAM, Aerosol Sci. and Technol., 40, 118-127, 2006.

Schell, B.: Die Behandlung sekundrer organischer Aerosole in einem komplexen Chemie-Transport-Modell, Ph.D. thesis, Univ. Kln, 2000.

Schell, B., Ackermann, I., Hass, H., Binkowski, F., and Ebel, A.: Modeling the formation of secondary organic aerosol within a comprehensive air quality model system, J. Geophys. Res., 106, $28275,2001 \mathrm{a}$.

Schell, B., Ackermann, I., Hass, H., Binkowski, F., and Ebel, A.: Modeling the formation of secondary organic aerosol within a comprehensive air quality model system, J. Geophys. Res., 106, 28 275-28 293, 2001b.
Seigneur, C.: Current status of air quality modeling for particulate matter, J. Air Waste Manage. Assoc., 51, 1508-1521, 2001.

Seinfeld, J. and Pandis, S.: Atmospheric chemistry and Physics, Wiley-interscience, 1998.

Sportisse, B., Debry, E., Fahey, K., Roustan, Y., Sartelet, K., and Tombette, M.: PAM project (Multiphase Air Pollution): description of the aerosol models SIREAM and MAM, Tech. Rep. 200608, CEREA, available at http://www.enpc.fr/cerea/polyphemus, 2006.

Spracklen, D., Pringle, K., Carslaw, K., Chipperfield, M., and Mann, G.: A global off-line model of size-resolved aerosol microphysics: I. Model development and prediction of aerosol properties, Atmos. Environ., 5, 2227-2252, 2005.

Stockwell, W., Kirchner, F., Kuhn, M., and Seefeld, S.: A new mechanism for regional atmospheric chemistry modeling., J. Geophys. Res., 95, 16343-16367, 1997.

Strader, R., Gurciullo, C., Pandis, S., Kumar, N., and Lurmann, F.: Development of gas-phase chemistry, secondary organic aerosol and aqueous-phase chemistry modules for PM modeling, Tech. rep., STI, 1998.

Vehkamki, H., Kulmala, M., Napari, I., K.E.J., L., Timmreck, C., Noppel, M., and Laaksonen, A.: An improved parameterization for sulfuric acid-water nucleation rates for tropospheric and stratospheric conditions, J. Geophys. Res., 107, 4622, 2002.

Verwer, J., Spee, E., Blom, J., and Hundsdorfer, W.: A second order Rosenbrock method applied to photochemical dispersion problem, SIAM J. SCI. COMPUT., 20, 1456-1480, 1999.

Wexler, A., Lurmann, W., and Seinfeld, J.: Modelling urban and regional aerosols -I. model development, Atmos. Environ., 28, 531-546, 1994.

Whitby, E. and McMurry, P.: Modal Aerosol Dynamics Modeling, Aerosol Science and Technology, 27, 673-688, 1997.

Zhang, Y., Seinfeld, J., Jacobson, M., Clegg, S., and Binkowski, F.: A comparative review of inorganic aerosol thermodynamic equilibrium modules: similarities, differences and their likely causes, Atmos. Environ., 1998.

Zhang, Y., Pun, B., Vijayaraghavan, K., Wu, S., Seigneur, C., Pandis, S., Jacobson, M., Nenes, A., and Seinfeld, J.: Development and application of the Model of Aerosol Dynamics, Reaction, Ionization and Dissolution (MADRID), J. Geophys. Res., 109, 2004. 